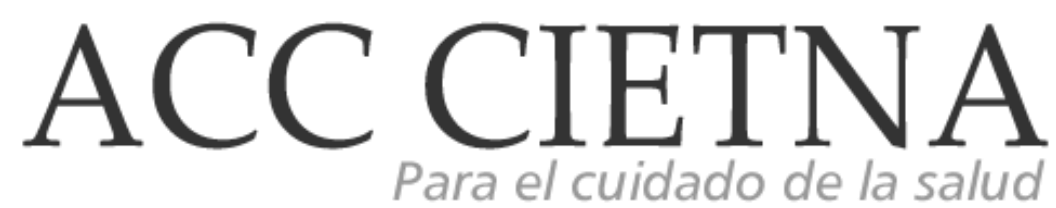

https://doi.org/10.35383/cietna.v6i2.268

\title{
Prácticas culturales en la estimulación temprana al lactante menor
}

\section{en una zona andina}

\section{Bustamante Llatas Jessica Paola', Gordillo Julón Miguel Amberly², Díaz Manchay Rosa Jeuna ${ }^{3}$, Rodríguez Cruz Lisseth Dolores ${ }^{4}$, Tejada Muñoz Sonia ${ }^{5}$}

\begin{tabular}{l}
\hline INFORMACIÓN DEL ARTÍCULO \\
\hline Historia del artículo: \\
Recibido el 27 de septiembre de 2019 \\
Aceptado el 21 de noviembre de 2019 \\
\hline Palabras clave: \\
Prácticas culturales \\
Estimulación \\
Familia \\
Lactante \\
Enfermería \\
Zona andina
\end{tabular}

\section{RESUMEN}

Introducción: La estimulación temprana es importante para el desarrollo integral del niño, siendo los padres los principales responsables; sin embargo, en las zonas de extrema pobreza como los andes del Perú, no se cuentan con materiales, ni el tiempo para hacerlo. Objetivo: Describir las prácticas culturales que hace la familia para estimular al lactante menor que vive en una zona andina del Perú. Métodos: Estudio cualitativo etnográfico ejecutado en los hogares de 15 familias que viven en un Distrito de Cajamarca, Perú; los datos se recolectó mediante la observación participante y la entrevista. Resultados: a) El juego, los juguetes y uso de la naturaleza en la estimulación temprana, b) Participación de hermanos mayores en la estimulación temprana. Conclusión: Los padres se dedican a las actividades agrícolas y crianza de animales, esto conlleva a que los hermanos mayores se responsabilicen del cuidado y por ende de la estimulación y el desarrollo psicomotor del niño (a) menor; además, no cuentan con la economía para comprar juguetes modernos, pero utilizan la naturaleza y sus propios recursos para la estimulación.

\footnotetext{
'Licenciado en Enfermería. Establecimiento de salud Pedregal Grande, Piura, Perú.

¿Licenciado en Enfermería. Puesto de Salud Pampa la Rioja, Cajamarca, Perú.

${ }^{3}$ Doctor en Ciencias de Enfermería. Especialista en salud familiar y comunitaria. Docente de la Universidad Católica Santo Toribio de Mogrovejo, Chiclayo, Perú. Teléfono: 990003610. Email: rdiaz@usat.edu.pe. ORCID: https://orcid.org/0000-0002-2333-7963 ${ }_{4}^{4}$ Mtro. en Enfermería. Docente de la Escuela de Enfermería de la Universidad Católica Santo Toribio de Mogrovejo Chiclayo, Perú. Teléfono: 991493120. Email: Irodriguez@usat.edu.pe. ORCID: https://orcid.org/0000-0003-1742-9498

${ }^{5}$ Doctora en Enfermería. Docente de la Universidad Nacional Toribio Rodríguez de Mendoza, Chachapoyas, Perú. Email: cieloceleste120@hotmail.com. ORCID: https://orcid.org/0000-0002-1181-8540
} 
Cultural practices in early stimulation to the minor infant in an andean area

ABSTRACT

\section{Keywords:}

Cultural practices

Stimulation

Family

Infant

Nursing

Andean area
Introduction: Early stimulation is important for the integral development of the child, the parents being the main responsible; however, in areas of extreme poverty such as the Andes of Peru, there are no materials or time to do so. Objective: Describe the cultural practices that the family does to stimulate the infant that lives in an Andean area of Peru. Methods: Qualitative ethnographic study carried out in the homes of 15 families living in a District of Cajamarca, Peru; Data was collected through participant observation and interview. Results: a) Play, toys and use of nature in early stimulation, b) Participation of older siblings in early stimulation. Conclusion: The parents are engaged in agricultural activities and animal husbandry, this leads to the older brothers being responsible for the care and therefore the stimulation and psychomotor development of the minor child; In addition, they do not have the economy to buy modern toys, but they use nature and their own resources for stimulation.

\section{Introducción}

Se calcula que un 43\% -249 millones- de los niños menores de 5 años en países de bajos y medianos ingresos corren un alto riesgo de sufrir menoscabo en su desarrollo debido a la extrema pobreza y el retraso en el crecimiento'. Esto es preocupante, pues la primera infancia que comprende entre la concepción y el inicio de la educación escolar brinda una oportunidad decisiva y única de influir en el desarrollo del cerebro de los niños; durante este periodo de la vida, el cerebro crece con asombrosa rapidez; las conexiones se forman a una velocidad que no volverá a repetirse en la vida, lo que determina $y$ afecta profundamente el desarrollo cognitivo, social y emocional del niño, influyendo en su capacidad para aprender, resolver problemas y relacionarse con los demás ${ }^{2}$.

De este modo, el primer año de vida se caracteriza por grandes cambios de crecimiento, diferenciación, adquisición y perfeccionamiento de funciones; por lo que resulta importante potenciar el desarrollo del niño a través de la estimulación temprana ofertando estímulos y aprendizaje de manera variada y abundante; pero están sometidas a la acción de factores biológicos y sociales que pueden alterar el desarrollo, e incluso retrasarlo ${ }^{3,4}$. La falta de estimulación en el primer año de vida del niño conlleva a tener consecuencias en el desarrollo de sus habilidades motoras, cognitivas, lingüísticas y sociales.

Ante lo mencionado, la estimulación temprana hace uso de experiencias significativas en las que intervienen los sentidos, la percepción y el gozo de la exploración, el descubrimiento, el autocontrol, el juego y la expresión artística; no busca exponer al niño a una serie de actividades sino, por el contrario, hacer que el niño las genere y las construya. Este principio modifica sustancialmente el papel del adulto, quien pasa a convertirse en un facilitador de experiencias 5 . Sin embargo, la madre y el padre cuentan con muy poco tiempo para 
compartir con sus hijos, pero deben generar situaciones de juego compartido entre los padres y el niño, en un ambiente cálido y estimulante que favorezca las manifestaciones de afecto ${ }^{5}$.

En un estudio se reportó, que los niños en zonas urbanas gozan de mayores niveles de estimulación en sus hogares que los niños de zonas rurales. No obstante, se evidencia bajos niveles en comunicación, en el desarrollo de vocabulario de los niños de zonas urbanas como rurales ${ }^{6}$. En otro estudio, identificaron que el rol de la madre se basa en la satisfacción de las necesidades básicas del niño: alimentación, higiene y cuidado de su salud a través del cumplimiento del calendario de vacunación; y en un segundo plano fomentan su desarrollo emocional y social, y es, en el día a día que va descubriendo los logros y potencialidades de su hijo?.

Con respecto a la estimulación temprana el profesional enfermero utiliza más la tarjeta de control del niño, y va explicando a las madres de acuerdo a su edad lo en casa debe enseñarle a su niño y así estimular las diferentes áreas del desarrollo psicomotor a través del juego. De esta manera la madre es la responsable de la estimulación, aunque en algunos zonas los hermanos mayores se encargan de las actividades de estimulación temprana (cognitivo, lingüístico, motor y social), y muchas veces a pesar de la pobreza y ciertas prácticas culturales logran un desarrollo psicomotor favorable del niño menor de un año que vive en las zonas altoandinas.

Objetivo: Describir las prácticas culturales que hace la familia para estimular al lactante menor que vive en una zona andina del Perú.

\section{Metodología}

Investigación cualitativa con enfoque etnográfico ${ }^{8}$. Los investigadores observaron los materiales y las actividades que realizabas los miembros de las familias andinas para estimular al lactante menor.
La muestra se determinó por la técnica de saturación y redundancia, siendo 15 madres de familia con niños lactantes menores que se atienden en un centro de salud de Cajamarca, naturales de esa zona, edad promedio de 22 años, con estudios de secundaria completa, eran amas de casa. La identidad de los sujetos fue protegida con códigos: M1 ...M15.

Los datos fueron recolectados mediante la observación participante y la entrevista etnográfica, que fueron elaborados por los investigadores y validados por juicio de expertos. Se realizaron cuatro visitas, con una duración promedio de cuatro horas cada una. La entrevista se realizó en la segunda visita, en un tiempo aproximado de 25 minutos. Asimismo, la información fue verificada y revisada por los sujetos de estudio para corroborar si están de acuerdo con lo manifestado.

Se usó el análisis temático para procesar los datos recolectados y fue de manera manual, desarrollando cuatro fases $9,10:$ a) En el análisis del dominio se identificaron elementos en los discursos y la observación que tengan significado cultural, b) En el análisis taxonómico se agruparon por semejanza los dominios culturales, c) En el análisis componencial se buscó componentes de significado cultural, patrones de contrastes y de similaridad y d) En el análisis de temas se buscó dimensiones de contraste entre los términos, lo que permitió obtener dos temas culturales.

Los participantes en el estudio no fueron expuestos a experimentación, no se les causó ningún tipo de daño; se protegió su identidad con códigos y participaron de manera voluntaria mediante la firma del consentimiento informado. La investigación fue ejecutada previa aprobación del Comité de Ética de la Facultad de Medicina de la Universidad Católica Santo Toribio de Mogrovejo, y con la debida autorización del director del establecimiento de salud. 


\section{Resultados}

a) El juego, los juguetes y uso de la naturaleza en la estimulación temprana

Las madres refieren que compran algunos juguetes, pues consideran que el juego es importante para que los niños socialicen con otros niños, además les va a ayudar a desarrollar capacidades cognitivas cuando estén en la escuela. Además utilizan materiales del hogar y hasta la misma naturaleza para estimular las diferentes áreas del desarrollo psicomotor.

"El juego es importante para que en la escuela se relacione con sus compañeros. Le hacemos jugar con pelotas, carritos, cubos, rueda de colores... conversamos y le enseño a decir palabras (papá, mamá). (M1, M6)

"Para que sea inteligente su papá le compró unas torres con círculos...lo dejo en el piso con una manta para que gatee y juegue...le dejo arrancar hojitas con sus dedos..." (M15)

"...le hago sus ejercicios según lo que la enfermera me enseño y está en la tarjeta de control para que aprenda más rápido en la escuela $y$ se lleven bien con sus compañeros...nos dice lo que debe lograr para el próximo control...también nos dijo que nos demos tiempo para jugar y hacer reír al bebé, pero no hay tiempo..." (M2, M9)

"Se observó que algunas madres llevan a su bebé en una manta envuelta en su espalda y la madre va haciendo sus quehaceres del hogar, le va conversando... Io hacen gatear al niño en el suelo que es de tierra, luego le lavan sus manos...a veces tienden una manta y le colocan juguetes y los hermanos mayores juegan con el bebé... " (Diario de campo)

Ente los juguetes más comunes que utilizan tenemos sonajas, pelotas, muñecas, y usan materiales del hogar, platos de plásticos, incluso la naturaleza como más adelante se detalla; por lo bajos recursos económicos en el que viven, no invierten dinero en juguetes.

Las madres reconocen que tienen poco tiempo para estimular al niño, por las múltiples tareas que desempeñan en la agricultura y la ganadería. Por otro lado, la enfermera educa con que materiales pueden estimular las madres a sus niños en casa. Otro aspecto importante es que ellos utilizan la naturaleza para estimular al niño.

"...le enseñamos como se llaman los animales: el pollo, el chancho, la gallina, el perro, les enseñamos los colores con las plantas, las flores; enseñamos a contar los árboles... incluso con los cerros le enseñamos a diferenciar cual es más grande...utilizamos la naturaleza y lo que tenemos en nuestra casa..." (M3, M5, M10)

"Se observa que las madres enseñan con los animales del hogar le van diciendo cual es la vaca, el toro, la gallina, los perros, gatos y el caballo... les enseñan los sonidos de los animales por ejemplo la madre le dice al niño como hace la vaca..." (Diario de campo)

Las madres hacen uso de la naturaleza y estimulan a sus hijos hacen con las plantas, flores, animales y hasta los cerros que les rodea; y van mostrando y enseñando los colores, el tamaño, entre otros aspectos.

\section{b) Participación de hermanos mayores en la estimulación temprana}

Las madres refieren que no dedican mucho tiempo a sus hijos para jugar porque no tienen tiempo, por estar en su chacra y otras madres refieren que todo el día están trabajando y ya no tienen el tiempo necesario para sus niños. Los hermanos mayores asumen la responsabilidad de jugar con el niño.

“...no tenemos tiempo para hacer jugar a los niños, pero sus hermanos mayores nos ayudan a cuidar al niño más pequeño $y$ juegan con ellos, todos nos apoyamos... les 
digo a sus hermanitos que no le vayan a dar cosas muy pequeñas porque lo llevan a la boca y se vaya a ahogar..." (M3, M5, M10)

"...Quien lo hace jugar son más sus hermanos, lo hacen jugar con sus carritos, sus peluches, ellos se dedican más a cuidar a sus hermanitos... no hay mucho tiempo porque más estamos en la chacra por eso no tenemos mucho tiempo..." (M12)

“... su papá juega poco con el más pequeño, debido a que se va a trabajar y solo viene por las tardes a almorzar y se va otra vez al trabajo, llega cansado y se va a dormir, pero a veces juega un ratito, aunque más me dedica a la chacra y al ganado..." (M12)

"...Se observó que cuando sale a la chacra lo deja al cuidado de los hermanos mayores...los hermanos le dan su biberón y lo hacen jugar con sonajas, tapas de gaseosas, platos de plásticos, cuando entran los pollos a la casa, le dicen entro el pio, pio..." (Diario de campo)

Asimismo, el padre, no se da tiempo para jugar con el bebé, ya que trabajo todo el día en la chacra y con el ganado. Se observa que cuando las madres tienen que salir a trabajar o están ocupadas los hermanos mayores se encargan del cuidado del niño por lo que aprovechan de una u otra manera hacerlo jugar al niño.

\section{Análisis y discusión}

La familia es la primera escuela del pequeño, ya que él imita lo que ve a su alrededor, tanto las acciones y formas de expresarse por parte de los que lo rodean, hasta su forma de pararse o vestir. Por tal motivo, es necesario estar consciente de la importancia que tiene la educación del infante, y de lo que probablemente puede aprender dentro del hogar. La presencia de los padres a lo largo del crecimiento del niño es de suma importancia, ya que cuando uno de los dos no está presente, es posible que el infante experimente dificultades en su desarrollo ${ }^{11}$. Garcia ${ }^{12}, \operatorname{Vargas}^{13}, \mathrm{Meza}^{14}$, en sus investigaciones consideran que los padres adquiere una singular importancia, ellos son los más cercanos educadores de los niños, los principales transmisores de la experiencia; de tal modo que la estimulación temprana comienza a partir del aprendizaje familiar; además, los padres asumen en relación con la salud, la nutrición, la importancia de los ambientes físico y social, y las oportunidades de estimulación y aprendizaje de los hijos.

En este estudio se demuestra que, en las familias andinas, sus integrantes colaboran no solo en el crecimiento del lactante menor, sino también en la estimulación mediante el juego. La mayoría de las madres reconocen la importancia del juego para estimular y que el niño adquiera mayor desarrollo en sus diferentes áreas como son motora, cognitiva y afectivo-social. Además, el juego en los niños/as tiene propósitos educativos y también contribuye en el incremento de sus capacidades creadoras, por lo que es considerado un medio eficaz para el entendimiento de la realidad.

El juego es una actividad inherente del ser humano; es la primera y la principal actividad por la que nos comunicamos con los demás, observamos y exploramos la realidad que nos rodea, establecemos relaciones con los objetos ${ }^{15}$. Uno de los aspectos más importantes de la vida de un niño es jugar; el juego es excelente para reducir el estrés y para liberar tensiones ${ }^{16}$. Perez ${ }^{17}$, es su estudio encontró que a través del juego el niño aprende a diferenciar los atributos de dos mundos diferentes, la fantasía y la realidad; en un primer momento, jugar y aprender quedan integrados, ya que a través del jugar el niño desplegará el lenguaje y construirá su universo simbólico.

Para jugar es necesario tener disponible juguetes apropiados para su edad. Los juguetes son un medio indispensable para que el niño logre su 
óptimo desarrollo e impedir que las actividades se consideren como "algo sin importancia" o "una pérdida de tiempo". La selección de un juguete dependerá de la edad cronológica y mental del niño, así como la condición socioeconómica de la familia. La elección de un juguete debe tener una finalidad y no debe servir para cumplir un capricho del niño o de los padres. Los niños no necesitan disponer de juguetes perfectos, si no que habrá que dejarle espacio y posibilidad de crear y disfrutar con sus propios jugos ${ }^{18}$. La finalidad es que las madres estimulen a sus niños a través del juego, no solamente con juguetes industrializados, sino que fabriquen sus propios juguetes de acuerdo con sus posibilidades y así jugar con su niño desde muy pequeño para evitar algún trastorno en su desarrollo.

Al respecto en este estudio, los juguetes más comunes que utilizan son las sonajas, pelotas, muñecas, carritos, según sexo del niño, se observa que los padres no invierten en comprar juguetes modernos o más tecnológicos, pues son de bajos recursos económicos; sin embargo, usan materiales del hogar como utensilios, maderas, platos de plásticos, e incluso usan su ecosistema para que el desarrollo cognitivo del niño.

Lo mencionado se corrobora con el estudio de Araujo y Rubio ${ }^{5}$, donde exploraron la disponibilidad de materiales de juego en los hogares por tipo de juguetes para tocar música, para armar o construir, para pintar, para el juego de roles, para aprender formas o a contar; encontraron que tanto en zonas urbanas como rurales, los juguetes más comunes son aquellos que requieren mucho movimiento físico (por ejemplo, pelotas) y los que sirven para el juego de roles. Mientras, Martinez, Vera, Tánori16, es su investigación con respecto a la estimulación del niño en el hogar, evaluaron la dimensión provisión de materiales de juego obtuvieron que los juguetes que los niños tienen son adecuados para su edad; estos juguetes eran carritos y muñecas, principalmente, los padres reportaron platicar con el niño durante los quehaceres domésticos. El ambiente común normal es el del propio hogar; es allí donde se debe brindar la estimulación temprana puesto que se aprovecha los momentos de atención habitual y las situaciones que vive el niño en interrelación con quienes los rodea ${ }^{19}$. Aspecto que en este estudio se revela, puesto que la madre mientras hace sus quehaceres del hogar, va conversando con el niño, y explicando los materiales que hay alrededor.

En este estudio las madres reconocen que la enfermera les enseña y motiva a que utilicen la naturaleza para estimular al niño, así hacen uso de los diferentes recursos que tiene en su alrededor como los animales y las plantas. Se observó que las madres enseñan a sus hijos a jugar con el medio ambiente que les rodea; las familias desde muy pequeños les van enseñando como se llaman los animales, o también les van haciendo diferenciar entre los árboles al más pequeño y al grande.

Al respecto Bustamante 20 , establece que el juego en la naturaleza está relacionado con probabilidades de desarrollar comportamientos ambientales adecuados; el aire libre puede generar en los niños respeto hacia la naturaleza, además el acercamiento a la naturaleza fomenta la experiencia de sus sentidos vitales, del tacto, el movimiento y el equilibrio, se favorecen las redes y conexiones internas que beneficiarán la óptima maduración biológica. Por otro lado, Torres, Alcántara y Mora ${ }^{21}$, manifiestan que la naturaleza ofrece una cantidad tan elevada de estímulos que el contacto con ella hace que el niño se encuentre en un espacio abierto, con sensación de libertad, con capacidad de moverse libremente, de observar los procesos que ocurren, y eso es fundamental para el desarrollo de sus habilidades de movimiento, pero también un estímulo para sus 
neuronas, para sus emociones y para su aprendizaje.

Se observó durante el tiempo de convivencia que tuvimos con las madres andinas, que son pocas la que dedican su tiempo a su niño, debido a su deberes que tiene que realizar durante el día; como es el cocinar, ir a la chacra, dar de comer a su animales y atender a su esposo; esto hace que la madre de familia no dedique el tiempo necesario, es por ello que sus hermano menores son los que juegan con ellos, a veces juegan en el gras tienden una manta en donde colocan al niño con sus juguetes ya sean carritos, cubos o muñecas si es niña, son ellos que se dedican hacer jugar a sus hermanitos pequeños. Además, la enfermera educa con qué materiales o juguetes que pueden estimular las madres a sus niños en casa.

Cuando las madres tienen que salir a trabajar o están ocupadas los hermanos mayores se encargan del cuidado del niño por lo que aprovechan de una u otra manera hacerlo jugar al niño. Asimismo, las madres refieren que son sus hijos los mayores quienes están pendientes de sus hermanos pequeños. Según Urbano22, la madre es el mejor agente de la estimulación temprana, pues ella es quien puede dedicar atención afectiva natural cercana, personal y permanente a su hijo. Aunque en el estudio concluyó que la baja interacción padre-hijo y madre-hijo en la estimulación del niño en el hogar, puede estar relacionada con el hecho de que las prácticas de crianza heredadas a los padres por la familia y la comunidad están enfocadas a la motricidad gruesa ya la socialización y dejan de lado otros aspectos igualmente importantes en el desarrollo como las habilidades cognitivas yel lenguaje ${ }^{16}$.

Por lo tanto, la consejería que realiza el profesional de enfermería con la madre debe ser en un clima de confianza, que permita que las madres andinas entiendan acerca de la importancia de la estimulación y que no solamente esté involucrada la madre o el hermano mayor, sino que participen todos los miembros de la familia. Tienen que tener un espacio para jugar con sus niños y no solamente dedicarse a la crianza de sus animales y cultivo de sus plantas.

\section{Conclusiones}

En este estudio se rescata que las madres reconocen la educación sanitaria que brinda el personal de enfermería, utilizan la tarjeta de control del niño, y explican a las madres lo que debe enseñarle a su niño en el hogar; además enfatiza sobre la importancia del juego para el aprendizaje y el desarrollo psicomotor del niño. Sin embargo, los padres se dedican a las actividades agrícolas y crianza de animales en la mayoría del día para el sustento económico del hogar; por ello se valora la participación de los hermanos en la estimulación, son ellos quienes juegan con los más pequeños. Por otro lado, no cuentan con la economía para comprar juguetes modernos, pero utilizan la naturaleza, los animales, los árboles, los cerros para que aprendan a distinguir colores, formas y tamaños.

Por tanto, se recomienda que la enfermera valore las prácticas que tienen en el hogar antes de brindar la educación sanitaria sobre el uso de materiales y las actividades a realizar para estimular al niño menor. Es necesario hacer estudios más específicos de las prácticas culturales en cada etapa de vida en diferentes zonas de pobreza, e incluso fomentar talleres interdisciplinarios con apoyo del gobierno municipal para un mejor seguimiento al crecimiento y desarrollo psicomotor el niño menor de un año. 


\section{Bibliografía}

1. Organización Mundial de la Salud (OMS). Invertir en el desarrollo en la primera infancia es esencial para que más niños, niñas y comunidades prosperen, concluye la nueva serie de The Lancet. Página oficial de la Oficial Organización Mundial de la Salud [Internet] Washington. [Octubre 2016, consultado 2019]. Disponible en:

https://www.who.int/es/news-

room/detail/05-10-2016-investing-inearly-childhood-development-essential-tohelping-more-children-and-communitiesthrive-new-lancet-series-finds

2. Fondo de las Naciones Unidas para la Infancia (UNICEF). United Nations Plaza [Internet]. EEUU; 2017 [Actualizado 2017; consultado 2019].

Disponible

en:

https://www.unicef.org/spanish/publications /files/UNICEF_Early_Moments_Matter_for_Eve ry_Child_Sp.pdf?fbclid=IwAR3UOBXfFAdOanQ LmkUAVpen-

acNNRKaJvWiY3L3Q2Sjo 1 zNLZ3j6IRmao0

3. Rodríguez J. Consejería de enfermería sobre estimulación temprana en madres primerizas y desarrollo psicomotor de los niños menores de 1 año. centro de salud materno infantil el bosque. 2011. [consultado el 09 octubre 2017]. Disponible en: http://repositorio.upao.edu.pe/bitstream/up aorep/227/1/RODRIGUEZ_JAKELIN_CONSEJER IA_ESTIMULACION_TEMPRANA.pdf

4. Organización Mundial de la Salud. [sede web]. Ginebra: Suiza; 2016. [acceso 1 de Setiembre del 2017]. Disponible en: http://www.who.int/maternal_child_adolesce nt/topics/child/development/es/

5. Ordoñez L, Tinajera M. Estimulación Temprana. Edición MMXVI. Mostoles - Madrid - España: Poligono Industrial Arroyomolino; 2014. 600p
6. Araujo M, Rubio M. ¿A que juegan los niños peruanos? - Primeros Pasos. [Internet] Banco interamericano de desarrollo. Diciembre 2016[consultado abril 2019]. Disponible en: https://blogs.iadb.org/desarrolloinfantil/es/ninos-peruanos/

7. Chinchay T, De la Cruz T. Rol de la madre en el cuidado de crecimiento y desarrollo del lactante menor. Centro de salud Pitipo 2015. [tesis para licenciatura en internet] Chiclayo: Universidad Santo Toribio de Mogrovejo; 2015 [consultada 30 de abril 2019]80 p. Disponible en:http://tesis.usat.edu.pe/bitstream/usat/4 77/1/TL_ChinchayPachecoTania_DeLaCruzCa rbonelTeresa.pdf?fbclid=IwAROJbOmQAJOXfBgSmwTtCkbrLo4HcOxXFsLztmVkTgkDdB1w Muptxg8R94

8. Grove S, Gray J, Burns N. Investigación en enfermería. Desarrollo de la práctica enfermera basada en la evidencia. 6a ed. Barcelona: Elsevier; 2016. 532p

9. Do Prado $M$, De Souza $M$, Monticelli $M$, Cometto M, Gómez P. Investigación cualitativa en enfermería. Metodología y didáctica. Washington DC: Organización Panamericana de la Salud; 2013. 253p.

10. McCurdy D, Spradley J, Shandy D. The cultural experience: Ethnography in Complex Society. $2^{\mathrm{a}}$ ed. Estados Unidos de América: Waveland Pr Inc; 2005.

11. Bustamante S. Enfermería familiar: Principios de cuidado a partir del saber (in) común de las familias. Departamento de salud familiar y comunitaria. Facultad de Enfermería de la Universidad Nacional de Trujillo. Trujillo; 2006.

12. García, M. Aprendizaje al aire libre en educación infantil. 2016 [consultado el 20 octubre 2017]. Disponible en: http://www.ugr.es/ patrimonioeducativo/am bitos/socializacion/María\%20García\%20Aivar \%20TFG\%20educacion\%20naturaleza.pdf 
13. García, M. Aprendizaje al aire libre en educación infantil. 2016 [consultado el 20 octubre 2017]. Disponible en: http://www.ugr.es/ patrimonioeducativo/am bitos/socializacion/María\%20García\%20Aivar \%20TFG\%20educacion\%20naturaleza.pdf

14. Vargas J, Aran V. Importancia de la Parentalidad para el Desarrollo Cognitivo Infantil: una .Revisión Teórica. Revista Latinoamericana de Ciencias Sociales, Niñez y Juventud, 12 (1), pp. 171-186. [consultado el 20 octubre 2017]. Disponible en: http://www.redalyc.org/html/773/77330034 $010 /$

15. Meza, M. Nivel de conocimientos y prácticas sobre estimulación temprana que tienen las madres de niños menores de un año de edad, que acuden al servicio de crecimiento $y$ desarrollo C.S Villa San Luis, San Juan de Miraflores, 2013 [consultado el 20 octubre2017].Disponible en:http://cybertesis.unmsm.edu.pe/bitstrea m/cybertesis/3592/1/Meza_fm.pdf

16. Betancourt L, Rodríguez M, Gempeler J. Interacción madre-hijo, patrones de apego y su papel en los trastornos del comportamiento alimentario. Revista de Psicología. [sede web] Colombia; 2012. [acceso 10 de Octubre del 2017]. Disponible en: http://med.javeriana.edu.co/publi/vniversita s/serial/v48n3/8-INTERACCION.pdf

17. Martínez L,Vera J, Tánori J. Interacción padres e hijos, la estimulación en el hogar y el desarrollo del niño en una zona rural de pobreza extrema. [tesis de licenciatura en internet]. Hermosillo, Sonora. Universidad de Sonora; 2003[consultada 20 abril2019].71 p. Disponible en: https://www.researchgate.net/publication/2 95549045_Interaccion_padres_e_hijos_la_esti mulacion_en_el_hogar_y_el_desarrollo_del_ni no_en_una_zona_rural_de_pobreza_extrema
18. Pérez C. La importancia del juego y los juguetes para el desarrollo integral de los niños/as de educación infantil, 2011 [consultado el 19 octubre 2017]. Disponible en:http://www.anpebadajoz.es/autodidacta/ autodidacta_archivos/numero_9_archivos/c_ p_cordero.pdf

19. Santos J. La importancia del juego y el vínculo materno en la constitución psíquica del infante. 2016. [acceso 20 de Octubre del 2017]. 36P. Disponible en:https://www.colibri.udelar.edu.uy/bitstrea $\mathrm{m} / 123456789 / 10087 / 1 /$ Santos\%2c\%20Jimen a\%20de\%20los.pdf

20. Ramos $Y$, Ramos $V$, Tortoló $S$. Los juguetes como medio de desarrollo del niño de la primera infancia. Rev. Científica pedagógica. Atenas Vol. 1Nro. 33 - 2016. [acceso 20 de Octubre del 2017]. 11p. Disponible en: https://atenas.reduniv.edu.cu/index.php/ate nas/article/view/190/357

21. Bustamante M. Convirtiendo su potencial en una realidad: experiencias de madres que participan en talleres de estimulación temprana. Chiclayo, 2012 [acceso 20 de Octubre del 2017]. Disponible en: http://tesis.usat.edu.pe/bitstream/usat/376/ $1 /$ TL_Bustamante_Parraguez_MariaJose.pdf

22. Torres J, Alcántara J, Mora M. Trabajando el acercamiento a la naturaleza de los niños y niñas en el Grado de Educación Infantil. Crucial en la sociedad actual. Revista Eureka sobre Enseñanza y Divulgación de las Ciencias [internet] 2017 [consultado el 20 octubre 2017]. 14 (1), 258-270 Disponible en: http://www.redalyc.org/html/920/92049699 $019 /$

23. Urbano C. modulo para el control del crecimiento y desarrollo de la niña y niño según las normas del ministerio de salud. Lima: MINSA. 2008. 